\title{
Probing Quark Gluon Plasma by Heavy Flavors
}

\author{
Santosh K Das* and Jan-e Alam \\ Theoretical Physics Division \\ Variable Energy Cyclotron Centre, 1/AF, Bidhan Nagar, Kolkata 700064, India \\ E-mail: santosh@vecc.gov.in, jane@vecc.gov.in
}

\begin{abstract}
The drag and diffusion coefficients of charm and bottom quarks propagating through quark gluon plasma (QGP) have been evaluated within the framework of perturbative Quantum Chromodynamics (pQCD). Both radiative and collisional processes of dissipation are included in evaluating these transport coefficients. The dead cone as well as the LPM effects on radiative energy loss of heavy quarks have also been considered. The Fokker Planck equation has been solved to study the dissipation of heavy quarks momentum in QGP. The nuclear suppression factor, $R_{\mathrm{A} A}$ and the elliptic flow $v_{2}^{H F}$ of the non-photonic electrons resulting from the semi-leptonic decays of hadrons containing charm and bottom quarks have been evaluated for RHIC and LHC nuclear collision conditions. We find that the observed $R_{\mathrm{AA}}$ and $v_{2}$ at RHIC can be reproduced simultaneously within the PQCD framework.
\end{abstract}

The Seventh Workshop on Particle Correlations and Femtoscopy

September 20 - 242011

University of Tokyo, Japan

*Speaker. 


\section{Introduction}

The nuclear collisions at Relativistic Heavy Ion Collider (RHIC) and Large Hadron Collider (LHC) energies are aimed at creating a new state of matter - properties of which are governed by thermal quarks and gluons, such a phase of matter is called Quark-Gluon Plasma(QGP). The study of the properties of QGP is a field of high contemporary interest and the heavy quarks (HQ) namely, charm and bottom quarks play crucial roles in this endeavour. Because HQs (i) are produced in the early stage of the collisions and hence witness the entire evolution scenario of the matter, (ii) do not dictate the bulk properties of the matter (iii) their thermalization time is larger than light quarks and gluons and therefore, the propagation of HQs through a QGP may be treated as the interactions between equilibrium and non-equilibrium degrees of freedom. The Fokker-Planck equation (FPE) provides an appropriate framework for such studies. In the present work we evaluate the nuclear suppression factor and elliptic flow of heavy flavours by solving the FPE for the motion of charm and bottom quarks in QGP and contrast the theoretical results with the data to extract the properties of QGP.

\section{Formalism}

The evolution of HQs momentum distribution function while propagating through the QGP can be described by the FPE, which reads [1],

$$
\frac{\partial f}{\partial t}=\frac{\partial}{\partial p_{i}}\left[A_{i}(p) f+\frac{\partial}{\partial p_{j}}\left[B_{i j}(p) f\right]\right]
$$

where the kernels $A_{i}$ and $B_{i j}$ are given by

$$
\begin{aligned}
& A_{i}=\int d^{3} k \omega(p, k) k_{i} \\
& B_{i j}=\int d^{3} k \omega(p, k) k_{i} k_{j}
\end{aligned}
$$

The function $\omega(p, k)$ is given by

$$
\omega(p, k)=g_{j} \int \frac{d^{3} q}{(2 \pi)^{3}} f_{j}(q) v_{i j} \sigma_{p, q \rightarrow p-k, q+k}^{j}
$$

where $g_{j}$ is the statistical degeneracy, $f_{j}$ is the phase space distribution of the particle $j, v_{i j}$ is the relative velocity between the two collision partners and $\sigma$ denotes the cross section. For $|\mathbf{p}| \rightarrow \mathbf{0}$, $A_{i} \rightarrow \gamma p_{i}$ and $B_{i j} \rightarrow D \delta_{i j}$ where $\gamma$ and $D$ stand for drag and diffusion co-efficients respectively.

As mentioned before we include both the collisional and radiative processes for HQs dissipation in QGP. For the collisional processes [2, 3]: $g Q \rightarrow g Q$ and $q Q \rightarrow q Q$ and $\bar{q} Q \rightarrow \bar{q} Q$ are considered in evaluating the drag $\left(\gamma_{\text {coll }}\right)$ and diffusion $\left(D_{\text {coll }}\right)$ coefficients.

For the radiative loss the drag coefficient is defined through the relation,

$$
\frac{-d E}{d x}=\gamma_{r a d} p
$$


and use the Einstein relation $D_{r a d}=T M \gamma_{r a d}$ to obtain the diffusion coefficient, where $M$ is the HQ mass, $T$ is the temperature of the QGP. In the radiative process the dead cone and LandauPomerenchuk-Migdal (LPM) effects are included [2]. Although the collisional and radiative processes are not independent from each other, however, in the absence of any rigorous method, we add them up to obtain the effective drag coefficients, $\gamma_{e f f}=\gamma_{\text {rad }}+\gamma_{\text {coll }}$, and similarly the effective diffusion coefficient, $D_{\text {eff }}=D_{\text {rad }}+D_{\text {coll }}$. This is a good approximation for the present work because the radiative loss is large compared to the collisional loss. In evaluating the drag co-efficient we have used temperature dependent strong coupling, $\alpha_{s}$ [4]. The Debye mass, $\sim g(T) T$ is also a temperature dependent quantity used here to shield the infrared divergences arising due to the exchange of massless gluons. The FPE has been solved with these effective transport coefficients and the initial momentum distributions of HQs are taken from the proton-proton (pp) collisions [5] at RHIC and LHC energies.

The solution of the FPE are convoluted with the fragmentation functions [6] of the HQs to obtain the $p_{T}$ distribution of the $D$ and $B$ mesons. The transverse momentum spectra of the electrons originating from the decays: $D \rightarrow X e v$ and $B \rightarrow X e v$ have been obtained by using standard procedures [7].

$(2+1)$ dimensional relativistic hydrodynamics [8] with boost invariance [9] along the longitudinal direction has been used for the space time description of the of the flowing QGP. We have taken the Equation of State $(\mathrm{EoS})$ as $P=c_{s}^{2} \varepsilon$, where $P$ is the pressure, $\varepsilon$ is the energy density and $c_{s}$ is the velocity of sound in QGP. Variation of $R_{A A}$ with $c_{s}^{2}$ i.e. with EoS will be presented here. We have assumed the value of the initial temperature, $T_{i}=400 \mathrm{MeV}$ and thermalization time of the QGP, $\tau_{i}=0.2 \mathrm{fm} / \mathrm{c}$ for Au+Au collisions at RHIC. The corresponding values for $T_{i}$ and $\tau_{i}$ for LHC are taken as $700 \mathrm{MeV}$ and $0.08 \mathrm{fm} / \mathrm{c}$ respectively. It is expected that the central rapidity region of the system formed in nuclear collisions at RHIC and LHC energies is almost net baryon density free. Therefore, the equation governing the conservation of net baryon number need not be considered here.

The total amount of energy dissipated by a HQ in the QGP depends on the path length it traverses. Each parton traverse different path length which depends on the geometry of the system and on the point where its is created. The probability that a parton is produced at a point $\left(r, \phi^{\prime}\right)$ in a QGP of ellipsoidal shape depends on the number of binary collisions at that point which can be taken as:

$$
P\left(r, \phi^{\prime}\right)=\frac{1}{\mathscr{N}}\left(1-\frac{r^{2}}{R^{2}} \frac{\left(1+\varepsilon \cos ^{2} \phi^{\prime}\right)}{\left(1-\varepsilon^{2}\right)^{2}}\right) \Theta(R-r)
$$

and

$$
\mathscr{N}=\frac{1}{\pi R^{2}\left(1-\frac{1}{2} \frac{1+\varepsilon^{2} / 2}{\left(1-\varepsilon^{2}\right)^{2}}\right)}
$$

where $R$ is the nuclear radius and $\varepsilon$ is the (spatial) eccentricity of the ellipse. A parton created at $\left(r, \phi^{\prime}\right)$ in the transverse plane propagate a distance $L=\sqrt{R^{2}-r^{2} \sin ^{2} \phi^{\prime}}-r \cos \phi^{\prime}$ in the medium. We use the following equation for the geometric average of the integral which appear in the solution of the FPE [3] involving drag coefficient:

$$
\Gamma=\frac{\int r d r d \phi^{\prime} P\left(r, \phi^{\prime}\right) \int^{L / v} d \tau \gamma(\tau)}{\int r d r d \phi^{\prime} P\left(r, \phi^{\prime}\right)}
$$


where $v$ is the velocity of the propagating partons. Similar averaging has been performed for the diffusion co-efficient.

\section{Results}

In this section we contrast the theoretical results with the experimental data available for $\mathrm{Au}+\mathrm{Au}$ collisions at RHIC energies and also present theoretical results for LHC energy.

\subsection{The Nuclear Suppression}

The transverse momentum distribution of electrons from the heavy flavours produced in $\mathrm{p}-\mathrm{p}$ collisions can be estimated from the charm and beauty quarks distributions which provides initial condition to the FPE. The solution of FPE for the HQ transverse momentum distribution contains the effects of drag (quenching) on the HQ whereas the initial distributions of HQ does not contain any such effects, therefore the ratio of these two quantities, the nuclear suppression factor, $R_{A A}$ acts as a marker for the momentum degradation in the medium, which is observed experimentally through, $R_{A A}\left(p_{T}\right)$ defined as:

$$
R_{A A}\left(p_{T}\right)=\frac{\frac{d N^{e}}{d^{2} p_{T} d y} \mathrm{~A} u+A u}{N_{\text {coll }} \times{\frac{d N^{e}}{d^{2} p_{T} d y}}^{\mathrm{p}+p}}
$$

The experimental data from both the PHENIX and STAR collaborations [10,11] shows substantial suppression $\left(R_{A A}<1\right)$ for $p_{T} \geq 2 \mathrm{GeV}$ indicating significant dissipation of HQ energy in the QGP. The theoretical results describe the data reasonably (Fig. 1, left panel). In the right panel we display the results for LHC for two values of $c_{s}$. We have taken an equation of state with velocity of sound lower than the value corresponding to the Stefan-Boltzmann limit. Lower value of $c_{s}$ [12], which is reasonable for strongly interacting QGP, makes the expansion of the plasma slower enabling the propagating heavy quarks to spend more time to interact in the medium and hence lose more energy before exiting from the plasma.

\subsection{Elliptic Flow}

The elliptic flow, $v_{2}^{H F}$ is defined as:

$$
v_{2}^{H F}\left(p_{T}\right)=\langle\cos (2 \phi)\rangle=\frac{\left.\int d \phi \frac{d N}{d y d p_{T} d \phi}\right|_{y=0} \cos (2 \phi)}{\left.\int d \phi \frac{d N}{d y d p_{T} d \phi}\right|_{y=0}}
$$

In the left panel of Fig. 2 we contrast the experimental data obtained by the PHENIX [11] collaborations for $\mathrm{Au}+\mathrm{Au}$ minimum bias collisions at $\sqrt{s_{\mathrm{NN}}}=200 \mathrm{GeV}$ with theoretical results obtain in the present work. It is observed that the $v_{2}^{H F}$ first increases and reaches a maximum of about $8 \%$ then saturates for $p_{T}>2 \mathrm{GeV}$. We also find that the data can be reproduced well by including both radiative and collisional loss with $c_{s}=1 / \sqrt{4}$. In the right panel we display the variation of $v_{2}^{H F}$ with $p_{T}$ for LHC energies with $c_{s}=1 / \sqrt{4}$. The wider difference between the theoretical results and the experimental data indicates that weaker coupling of the high $p_{T} \mathrm{HQs}$ with the QGP may required to be included in the calculations. 

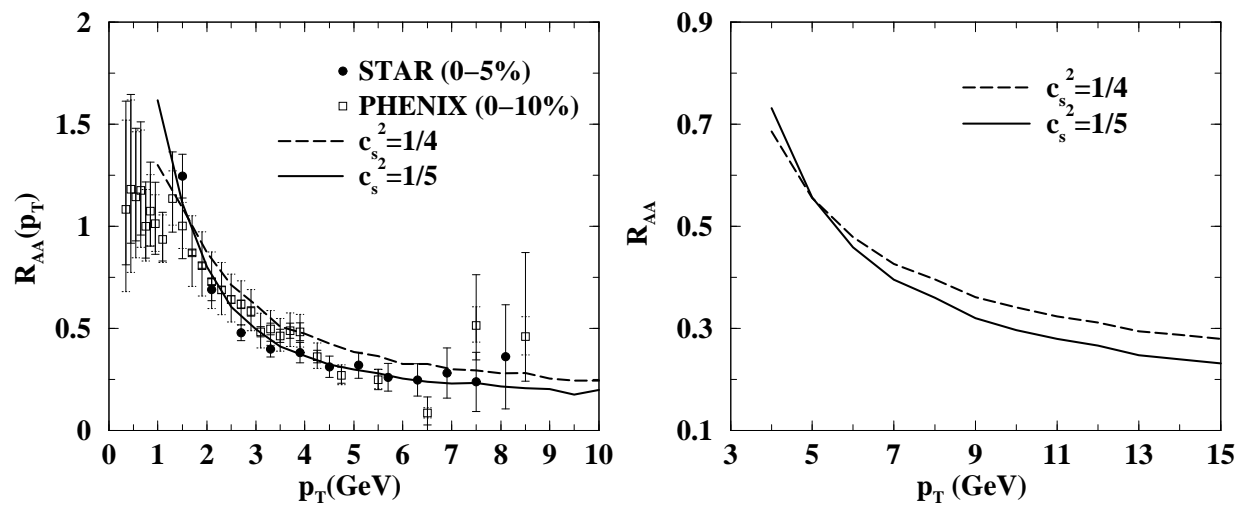

Figure 1: Left panel: variation of $R_{\mathrm{A} A}$ with $p_{T}$ obtained from the present work compared with the experimental data measured by STAR and PHENIX collaboration at $\sqrt{s_{\mathrm{N} N}}=200 \mathrm{GeV}$. The experimental data of STAR and PHENIX collaborations are taken from [10] and [11] respectively. Right panel: variation of $R_{A A}$ with $p_{T}$ for LHC.
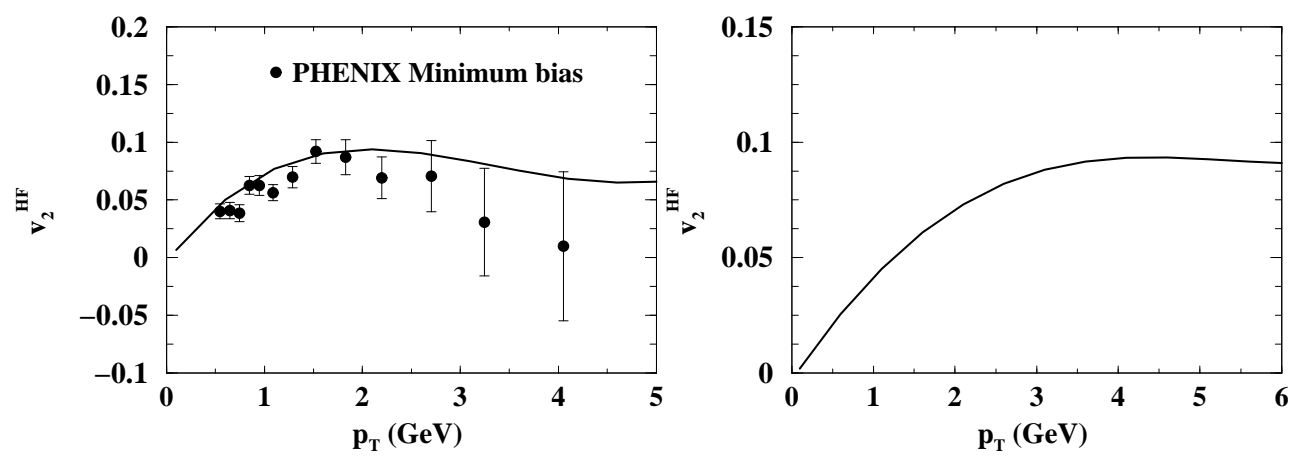

Figure 2: Left panel: variation of $v_{2}^{H F}$ with $p_{T}$ at the highest RHIC energy. Experimental data is taken from [11]. The value of the "effective" impact parameter, $b=10.2 \mathrm{fm} / \mathrm{c}$ for minimum bias Au+Au collision at $\sqrt{s_{\mathrm{NN}}}=200 \mathrm{GeV}$. Right panel: variation of $v_{2}^{H F}$ with $p_{T}$ for LHC.

\section{Summary and discussions}

In summary, we have simultaneously reproduced the measured nuclear suppression and elliptic flow of heavy flavours at RHIC energies within the framework of pQCD interactions of the nonequilibrated HQ with flowing QGP. We observe that the inclusion of the collisional and radiative processes of dissipation and use of non-ideal EoS are the key factors for the successful reproduction of the data. Our analysis admits the formation of QGP in Au+Au collisions at centre of mass energy, $\sqrt{s_{\mathrm{NN}}}=200 \mathrm{GeV}$ with initial temperature $\sim 400 \mathrm{MeV}$.

Some comments on the $R_{\mathrm{AA}}$ vis-a-vis $v_{2}^{H F}$ are in order here. The $R_{\mathrm{A} A}$ contains the ratio of $p_{T}$ distribution of the electrons resulting from $\mathrm{Au}+\mathrm{Au}$ to $\mathrm{p}+\mathrm{p}$ collisions, while the numerator contains the interaction of the HQs with the flowing QGP, such interactions are absent in the denominator. Whereas for $v_{2}^{H F}$ both the numerator and the denominator contain the interactions with the medium, resulting in some sort of cancellation. Therefore, $K$ factor which is sometimes used for 
the reproduction of the data may not be same for $R_{\mathrm{A} A}$ and $v_{2}^{H F}$.

Several theoretical attempts have been made to explain $R_{\mathrm{A} A}$ and $v_{2}$, where the role of hadronic matter has been ignored. However, to make the characterization of QGP reliable the role of the hadronic phase should be taken into consideration and its contribution must be subtracted out from the observables. Recently the drag and diffusion coefficients of hot hadronic medium consisting of pions, kaons and eta using open charm and beauty mesons as a probe have been evaluated $[13,14,15,16,17]$. It is observed that the magnitude of both the transport coefficients are significant, indicating substantial interactions of the heavy mesons with hot hadrons, which may have significant consequences of $R_{A A}$ and $v_{2}^{H F}$.

Acknowledgment: SKD is supported by DAE-BRNS project Sanction No. 2005/21/5-BRNS/2455.

\section{References}

[1] B. Svetitsky, Phys. Rev. D 37, 2484( 1988); H. van Hees, R. Rapp, Phys. Rev. C,71, 034907 (2005); G. D. Moore and D. Teaney, Phys. Rev. C 71, 064904 (2005); H. van Hees, M. Mannarelli, V. Greco and R. Rapp, Phys. Rev. Lett. 100, 192301 (2008); Y. Akamatsu, T. Hatsuda and T. Hirano, Phys. Rev. C 79, 054907 (2009); S. K Das, J. Alam and P. Mohanty, Phys. Rev. C 80, 054916 (2009); S. K Das, J. Alam, P. Mohanty and B. Sinha Phys. Rev. C 81, 044912 (2010); W. M. Alberico et al.,

Eur.Phys.J.C 71, 1666 (2011); S. Majumdar, T. Bhattacharyya, J. Alam and S. K. Das, Phys. Rev. C 84, 044901 (2011).

[2] S. K Das, J. Alam and P. Mohanty, Phys. Rev. C 82, 014908 (2010).

[3] S. K. Das and J. Alam, arXiv: 1008.2643 [nucl-th];

[4] O. Kaczmarek and F. Zantow, Phys. Rev. D, 71, 114510 (2005).

[5] M. L. Mangano, P. Nason and G. Ridolfi, Nucl. Phys. B 538, 282 (2002).

[6] C. Peterson et al., Phys. Rev. D 27, 105 (1983).

[7] M. Gronau et al. Nucl. Phys. B 123, 47 (1977); A. Ali, Z. Phys. C 1, 25 (1979).

[8] P. F. Kolb, J. Sollfrank and U. Heinz, Phys. Rev. C 62, 054909 (2000); P. F. Kolb and R. Rapp, Phys. Rev. C 67, 044903 (2003); P. F. Kolb and U. Heinz, nucl-th/0305084, J. Sollfrank, P. Koch and U. Heinz, Phys. Lett. B 252, 256 (1990) J. Sollfrank, P. Koch and U. Heinz, Z. Phys. C 52, 593 (1991).

[9] J. D. Bjorken, Phys. Rev. D 27, 140 (1983)

[10] B. I. Abeleb et al. (STAR Collaboration), Phys. Rev. Lett. 98, 192301 (2007).

[11] S. S. Adler et al. (PHENIX Collaboration), Phys. Rev. Lett. 98, 172301 (2007)

[12] S. Borsányi et al., J. High. Ener. Phys 1011, 077 (2010)

[13] S. Ghosh, S. K. Das, S. Sarkar and J. Alam, Phys. Rev. D(R) 84,011503(R) (2011)

[14] S. K. Das, S. Ghosh, S. Sarkar and J. Alam, arXiv: 1109.3359[hep-ph]

[15] M. Laine,JHEP 04, 124 (2011)

[16] M. He, R. J. Fries and R. Rapp, Phys. Let. B701, 445 (2002)

[17] L. Abreu, D. Cabrera, F. J. Llanes-Estrada and J. M. Torres-Rincon, arXiv:1104.3815 [hep-ph] 